\title{
Increased vaccine efficacy against tuberculosis of recombinant Mycobacterium bovis bacille Calmette-Guérin mutants that secrete listeriolysin
}

\author{
Leander Grode,1 Peter Seiler, ${ }^{1}$ Sven Baumann, ${ }^{1}$ Jürgen Hess, ${ }^{1}$ Volker Brinkmann,1 \\ Ali Nasser Eddine, ${ }^{1}$ Peggy Mann, ${ }^{1}$ Christian Goosmann, ${ }^{1}$ Silke Bandermann, ${ }^{1}$ Debbie Smith, ${ }^{2}$ \\ Gregory J. Bancroft, ${ }^{2}$ Jean-Marc Reyrat,, ${ }^{3}$ Dick van Soolingen, ${ }^{4}$ Bärbel Raupach, ${ }^{1}$ \\ and Stefan H.E. Kaufmann ${ }^{1}$

\begin{abstract}
${ }^{1}$ Max Planck Institute for Infection Biology, Berlin, Germany. ${ }^{2}$ Immunology Unit, Department of Infectious and Tropical Diseases, London School of Hygiene and Tropical Medicine, London, United Kingdom. ${ }^{3}$ INSERM, Faculté de Médecine Necker-Enfants Malades, Paris, France. ${ }^{4}$ National Mycobacteria Reference Laboratory, National Institute of Public Health and the Environment, Bilthoven, The Netherlands.
\end{abstract}

\begin{abstract}
The tuberculosis vaccine Mycobacterium bovis bacille Calmette-Guérin (BCG) was equipped with the membraneperforating listeriolysin (Hly) of Listeria monocytogenes, which was shown to improve protection against Mycobacterium tuberculosis. Following aerosol challenge, the Hly-secreting recombinant BCG $\left(b l y^{+} \mathrm{rBCG}\right)$ vaccine was shown to protect significantly better against aerosol infection with $M$. tuberculosis than did the parental BCG strain. The isogenic, urease C-deficient $b l y^{+}$rBCG ( $\triangle u r e C b l y^{+}$rBCG) vaccine, providing an intraphagosomal $\mathrm{pH}$ closer to the acidic $\mathrm{pH}$ optimum for Hly activity, exhibited still higher vaccine efficacy than parental BCG. $\Delta u r e C h l y^{+}$rBCG also induced profound protection against a member of the M. tuberculosis Beijing/W genotype family while parental BCG failed to do so consistently. Hly not only promoted antigen translocation into the cytoplasm but also apoptosis of infected macrophages. We concluded that superior vaccine efficacy of $\Delta u r e C h l y^{+}$rBCG as compared with parental BCG is primarily based on improved cross-priming, which causes enhanced $T$ cell-mediated immunity.
\end{abstract}

\section{Introduction}

Mycobacterium bovis bacille Calmette-Guérin (BCG) represents the most widely used viable vaccine for tuberculosis, with over 3 billion doses administered (1). General agreement exists that BCG can protect against, or at least ameliorate, severe forms of systemic tuberculosis in children, particularly meningitis. However, BCG's protective value against adult pulmonary tuberculosis ranges from little to none. Although approximately $95 \%$ of all tuberculosis cases occur in the developing world, several countries of eastern Europe, including Russia, have witnessed increasing incidences of tuberculosis in recent years, with an estimated 9 million new tuberculosis cases globally in 2002 (2). Worldwide estimated mortality ranges from 1.6 to 2.2 million lives per year, depending on whether the half-million individuals suffering coinfection with HIV and Mycobacterium tuberculosis, are added to the toll of HIV or tuberculosis.

Recent field studies identified a novel genotype family of $M$. tuberculosis, Beijing, or W, which is increasingly spreading over the globe. It has been proposed that under the selective pressure of mass BCG vaccination and broad-scale administration of tuber-

Nonstandard abbreviations used: BCG, Mycobacterium bovis bacille CalmetteGuérin; Hly, listeriolysin; rBCG, recombinant BCG; bly rBCG, Hly-secreting rBCG; $\Delta$ ureC $b y^{+}$rBCG, ureC-deficient $h y^{+}$rBCG; ureC, urease C.

Conflict of interest: L. Grode, S.H.E. Kaufmann, and J. Hess are the inventors of the rBCG strains, and they have exclusively licensed them through the Max Planck Society to Vakzine Projekt Management GmbH (VPM; Hannover, Germany). S.H.E. Kaufmann reports having received research grant support from VPM, and L. Grode is now an employee of VPM.

Citation for this article: J. Clin. Invest. 115:2472-2479 (2005).

doi:10.1172/JCI24617. culostatics, the population structure of M. tuberculosis is changing in favor of the selection of conserved genotypes such as Beijing with higher survival fitness for these conditions (3-5). This is particularly worrisome since this genotype family comprises a high proportion of multidrug-resistant strains, which are rapidly expanding globally $(6,7)$. In light of increasing incidences of multidrug-resistant $M$. tuberculosis strains, the urgent need for a more efficacious tuberculosis vaccine is no longer restricted to the developing world.

The preferred intraphagosomal location of M. tuberculosis and BCG in professional APCs - mostly macrophages and DCs - dictates trafficking of their antigens through the MHC II pathway, which results in preferential stimulation of CD4 T cells (8). Yet, MHC I-restricted CD8 T cells are known to contribute to immunity to M. tuberculosis (8). Recent evidence suggests that $M$. tuberculosis induces apoptosis in infected cells, which in turn results in the formation of vesicles carrying mycobacterial antigens that can be taken up by bystander APC (9). This process, termed cross-priming, not only promotes MHC I antigen processing and CD8 T cell stimulation but also bypasses immunosuppressive mechanisms imposed on the infected cell (10). BCG only induces weak apoptosis and CD8 $\mathrm{T}$ cell stimulation (9).

To improve access of mycobacterial antigens to the MHC I pathway, which would result in better CD8 T cell stimulation, we constructed an rBCG strain that secretes listeriolysin (Hly) of Listeria monocytogenes (11). The Hly enables L. monocytogenes to escape from the phagosomes of infected host cells (12). Hly has an acidic $\mathrm{pH}$ optimum of greater than 5.8 (13) while BCG modifies the phagosomal $\mathrm{pH}$ to remain neutral (14). To improve the $\mathrm{pH}$ 
A
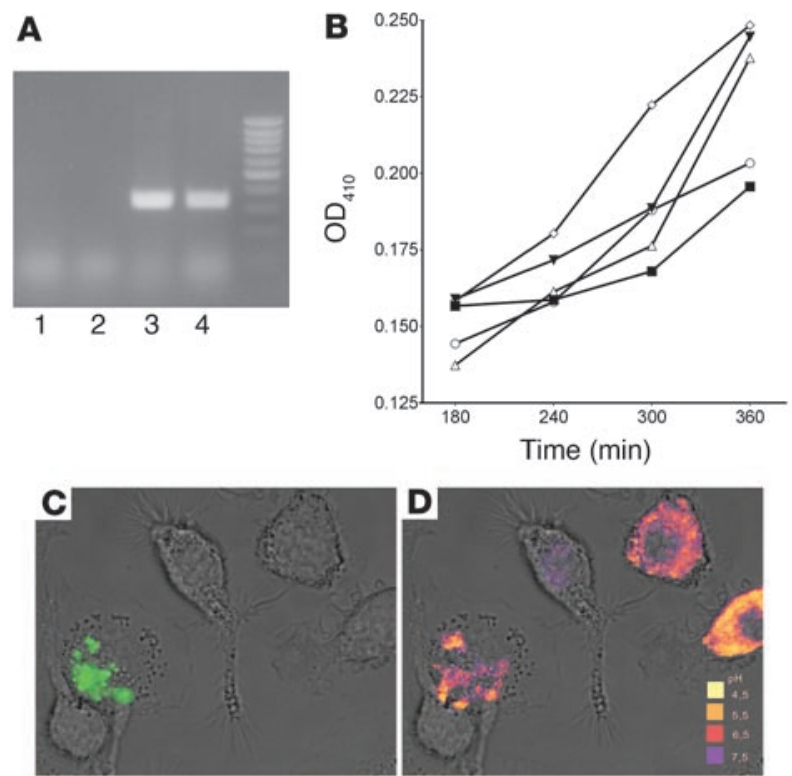

E
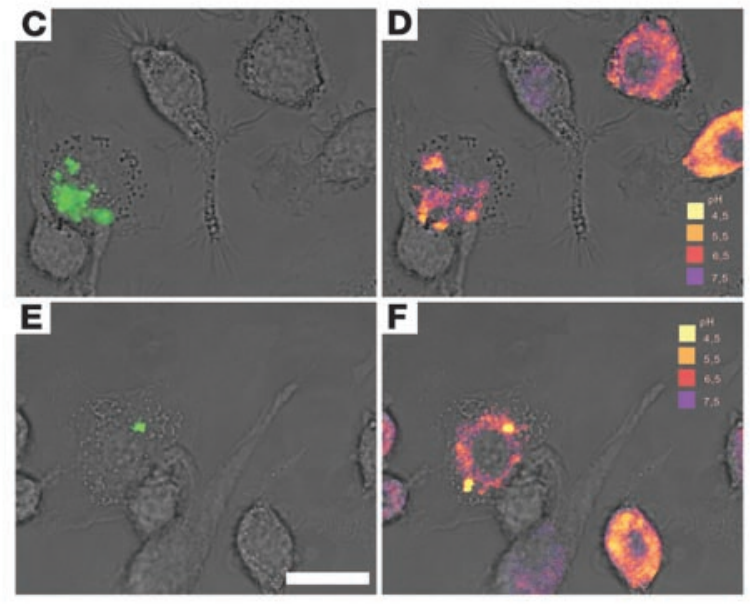

milieu for Hly activity, a urease C-deficient (ureC-deficient) Hlysecreting BCG-vaccine strain was constructed (15). Mycobacterial ureC plays a role in $\mathrm{pH}$ neutralization of the phagosome and thus inhibits maturation of the phagolysosome $(14,16)$. Here, we demonstrate that Hly-secreting recombinant BCG ( $b l y^{+}$rBCG) and ureC-deficient $b l y^{+}$rBCG $\left(\Delta u r e C h l y^{+}\right.$rBCG) mutant strains induce more efficacious protection against $M$. tuberculosis than does the parental BCG strain in a low-to-medium dose aerosolinfection mouse model.

\section{Results}

Generation and characterization of $\triangle u r e C h l y^{+} r B C G$. In order to generate a live vaccine that provides improved protection against $M$. tuberculosis, we equipped BCG with the membrane-perforating Hly of L. monocytogenes ( $h l y^{+} \mathrm{rBCG}$ ) (Figure 1A). To improve the $\mathrm{pH}$ milieu for Hly activity, a $\Delta u r e C h l y^{+} \mathrm{rBCG}$ strain that lacked ureC activity was constructed. As predicted, hemolytic activities of supernatants from $b l y^{+} \mathrm{rBCG}$ and $\Delta u r e C h l y^{+} \mathrm{rBCG}$ cultures against sheep red blood cells were considerably more intense than those seen with parental BCG (Figure 1B). To show whether absence of ureC allowed phagosomal acidification, the $\mathrm{pH}$ of mycobacterial phagosomes was determined by ratiometric analysis of dye-labeled phagosomes containing parental or $\Delta u r e C h l y^{+}$rBCG organisms. Macrophages were infected with DID-stained BCG strains. Phase contrast with fluorescence overlay is shown in Figure 1, C and E, while parts D and $\mathrm{F}$ show the same cells with $\mathrm{pH}$ pseudocolor. As expected, parental BCG faced an almost neutral pH (Figure 1, C and D) whereas Hly produced by $\Delta u r e C h l y^{+}$rBCG faced an acidic milieu between pH 4.5 and pH 5.5 (Figure 1, E and F). Similar effects were observed with parental BCG or $\Delta u r e C h l y^{+}$rBCG cova-

\section{Figure 1}

Hemolytic activity of and phagosomal acidification by $\Delta u r e C h l y^{+}$rBCG. (A) RT-PCR analysis of BCG-strains for Hly-secretion. Secretion of Hly was analyzed by RT-PCR using RNA from parental BCG (lane 1), rBCG $\Delta u r e C$ (lane 2), hly+ rBCG (lane 3), and sureC hly+ rBCG (lane 4). Shown is 1 out of 3 representative experiments. (B) Hemolysis by $h l y^{+}$rBCG and $\triangle$ ure C hly ${ }^{+}$rBCG but not parental BCG. Sheep red blood cells were incubated with $h / y^{+}$rBCG (open triangles), $\Delta u r e C h / y^{+}$ rBCG (closed triangles), parental BCG (closed squares), or L. monocytogenes (open diamonds), or remained untreated (open circles). At indicated time points, aliquots were taken and release of hemoglobin was determined by optical absorption as measurement for lysis of red blood cells. Shown is 1 representative experiment of 4 . (C-F) Primary murine macrophages were infected with parental BCG (C and $\mathbf{D})$ or $\Delta u r e C h l{ }^{+}$rBCG (E and F) for 2.5 hours. Images on the left show BCG stained with DID (signal shown in green). Images on the right show a pseudocolor representation of the fluorescence ratio in the blue and green channels with the Lyso Sensor Yellow/Blue dye. Images are merged with a black-and-white phase contrast image. Note that not all bacteria in each batch were stained due to the poor solubility of DID and its high affinity to the hydrophobic cell wall. Scale bar: $20 \mu \mathrm{m}$.

lently coupled to the pH-sensitive dye Cypher5 (data not shown). While parental BCG showed a weak fluorescence signal over the 60-minute recording period, $\Delta u r e C h l y^{+} \mathrm{rBCG}$ gained strong fluorescence intensity after uptake by macrophages, and bacteria outside of cells did not show changes in fluorescent signal.

Superior vaccine efficacy of $h l y^{+} r B C G$ and $\triangle$ ure $C$ bly $r B C G$ against $M$. tuberculosis laboratory strain H37Rv and clinical isolate of the Beijing/W genotype family. BALB/c mice were vaccinated (one single i.p. injection of $\left.10^{6} \mathrm{CFU}\right)$ with $b l y^{+} \mathrm{rBCG}, \Delta u r e C h l y^{+} \mathrm{rBCG}$, or parental BCG and aerosol challenged 120 days after vaccination with $M$. tuberculosis $\mathrm{H} 37 \mathrm{Rv}$ microorganisms. At this time point, BCG had been completely eradicated in vaccinated mice (verified by CFU analysis, data not shown). Vaccination with $h l y^{+}$rBCG and parental BCG considerably reduced $M$. tuberculosis load in the lungs of aerosol-challenged mice as compared with naive controls (Figure $2 \mathrm{~A}$ ). In the early (day 30) to intermediate (day 90) phase of infection, the course of $M$. tuberculosis $\mathrm{H} 37 \mathrm{Rv}$ infection did not significantly differ between the 2 vaccinated groups. However, at late stages (day 150) of infection, the M. tuberculosis H37Rv loads in the lung were significantly $(P=0.016)$ reduced by 1 order of magnitude in $h l y^{+}$rBCG-vaccinated mice as compared with mice in the parental BCG group.

Although both $\Delta u r e C h l y^{+}$rBCG and parental BCG protected against tuberculosis, protection evoked by $\Delta u r e C h l y^{+}$rBCG was substantially more successful than that evoked by parental BCG (Figure 2, B and C). This was true for infection with medium doses (200 CFU; Figure 2C) and low doses (30 CFU; Figure 2B) of $M$. tuberculosis. In the experiment shown in Figure 2B, protection led to an almost 1,000-fold reduction of the $M$. tuberculosis load in lungs as compared with naive mice at day 200 after challenge. Also, at earlier time points, enhanced protection was seen with $\Delta u r e C$ bly ${ }^{+}$rBCG as compared with parental BCG (Figure 2B).

An equally striking improvement was noted after challenge with the clinical genotype strain $M$. tuberculosis Beijing/W (Figure 3, A and B). The $\triangle$ ure C bly ${ }^{+} \mathrm{rBCG}$ strain significantly $(P<0.001$ at day $200)$ protected against the Beijing/W genotype strain in both lung and spleen over the entire 200-day period of observation $(P<0.05)$. Compared with vaccination with parental BCG, which virtually failed to protect against M. tuberculosis Beijing/W in this experi- 


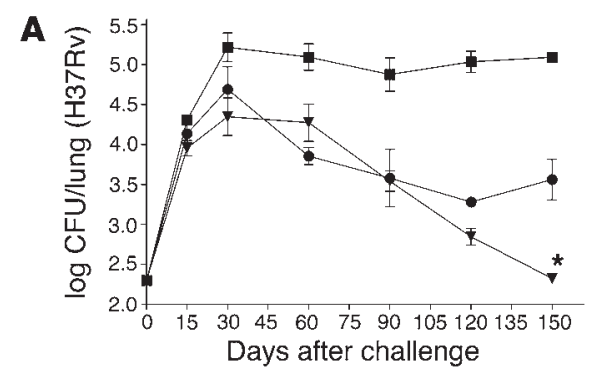

B
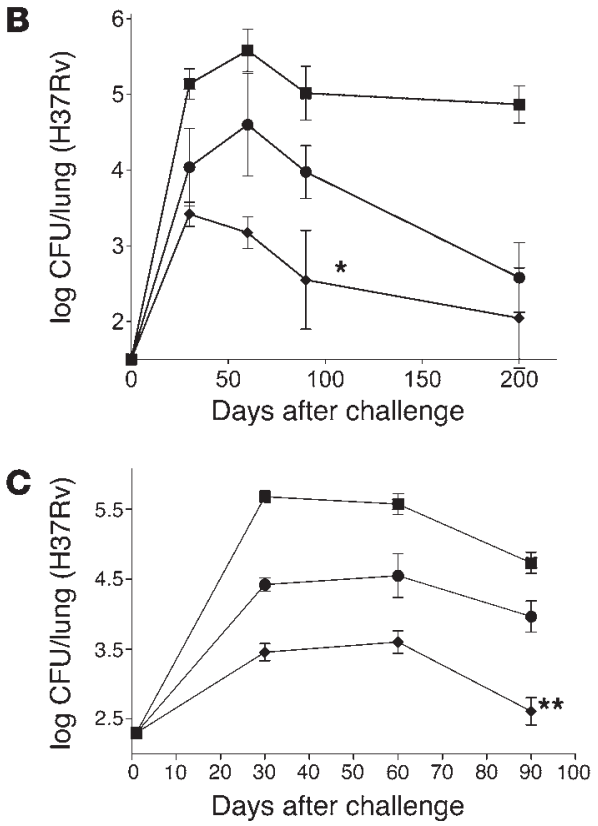

ment, vaccination with $\triangle u r e C b l y^{+} \mathrm{rBCG}$ reduced $\mathrm{CFU}$ in the lung by more than 100 -fold.

In vivo safety of $h l y^{+} r B C G$ and $\triangle u r e C h l y^{+} r B C G$. So that we could analyze the safety of the new $\mathrm{rBCG}$ vaccine strains in vivo at high doses, SCID mice were vaccinated i.v. with approximately $10^{8} \mathrm{CFU}$ (Figure 4A). At this high dose, SCID mice vaccinated with $b l y^{+}$ rBCG survived significantly longer (mean survival time, 65 days; $P=0.001$ ) than did SCID mice vaccinated with parental BCG (mean survival time, $<25$ days). The $\Delta u r e C h l y^{+}$rBCG-vaccinated mice survived even longer (mean survival time, 80 days; $P<0.001$ ) than mice vaccinated with parental BCG. Thus, in the SCID mouse model, virulence of the rBCG strains was substantially reduced as compared with parental BCG. In further support of the high safety of $h l y^{+}$

\section{Figure 3}

Greater protection against $M$. tuberculosis Beijing/W by vaccination with $\Delta u r e C h l y^{+}$rBCG. BALB/c mice were vaccinated with $\Delta u r e C h l y^{+}$ rBCG (diamonds) or parental BCG (circles) or left naive (squares). At day 120 after vaccination, mice were aerosol challenged with 200 CFU of $M$. tuberculosis Beijing/W. M. tuberculosis load in lung was determined at the indicated time points. Upper panel: lung, $P<0.001$ (Mann-Whitney $U$ test) for $\Delta u r e C h l y^{+}$rBCG compared with parental BCG at day 200 p.i.; and $P<0.05$ (Friedman test) for the whole observation period. Lower panel: spleen, $P<0.001$ (Mann Whitney $U$ test) for $\triangle$ ure $C h l y+$ rBCG compared with parental BCG at day 200 p.i. Shown is 1 representative experiment of 2 . The mean of results for 7 mice is shown ( \pm SEM).

\section{Figure 2}

Greater protection against $M$. tuberculosis aerosol challenge by vaccination with $h l y^{+} \mathrm{rBCG}^{+}$and $\Delta u r e C h l y^{+} \mathrm{rBCG}$. (A) BALB/c mice were vaccinated with $h l y^{+} \mathrm{rBCG}$ (triangles) or parental BCG (circles) or left naive (squares). At day 120 after vaccination, mice were aerosol challenged with $200 \mathrm{CFU}$ of $M$. tuberculosis H37Rv. $P=0.016$ (MannWhitney $U$ test) for $h l y^{+}$rBCG compared with parental BCG at day 150 after infection (p.i.). Shown is 1 representative experiment of 2. The mean of results from 7 mice is shown ( \pm SEM). (B and C) BALB/C mice were vaccinated with $\Delta u r e C h l y^{+} \mathrm{rBCG}$ (diamonds) or parental BCG (circles) or left naive (squares). At day 120 after vaccination, mice were aerosol challenged with $30 \mathrm{CFU}$ (B) and $200 \mathrm{CFU}$ (C) of M. tuberculosis H37Rv. M. tuberculosis load in lung was determined at the indicated time points. Shown are 2 representative experiments. ${ }^{*} P=0.0012$ and ${ }^{* *} P=0.001$ (Mann-Whitney $U$ test) for $\Delta u r e C h / y^{+}$ rBCG compared with parental BCG at day 90 p.i. The mean of results for 7 mice is shown ( \pm SEM).

rBCG vaccine candidates, CFUs in spleen and lung did not differ among BALB/c wild-type mice vaccinated with the 3 different BCG strains, and all 3 strains were cleared by a similar kinetics (data not shown). Similarly, CFU in spleen and lung of RAG1/- mutant mice after vaccination with $h l y^{+} \mathrm{rBCG}$ and $\Delta u r e C h l y^{+} \mathrm{rBCG}$ did not significantly differ from CFU of mice vaccinated with parental BCG (Figure 4B). Consistent with these findings, histological analysis of vaccinated mice did not reveal any alterations in gross pathology in lung, spleen, and liver of either immunocompetent BALB/c mice or RAG $1^{-/-}$mutant mice (data not shown).

Antigen translocation and apoptosis of host cells infected with bly ${ }^{+} \mathrm{rBCG}$. We wanted to gain deeper insight into the biological mechanisms responsible for superior vaccine efficacy of $\Delta u r e C b l y^{+}$rBCG. Two mechanisms can be envisaged, both depending on the capacity of Hly to perforate the phagosomal membrane. These mechanisms are not mutually exclusive. First, perforation of the membrane surrounding the mycobacterial phagosome facilitates translocation and subsequent MHC I loading of mycobacterial antigens $(11,17)$. Second, membrane perforation promotes leakage of phagolysosomal proteases such as cathepsins into the cytosol, which activate
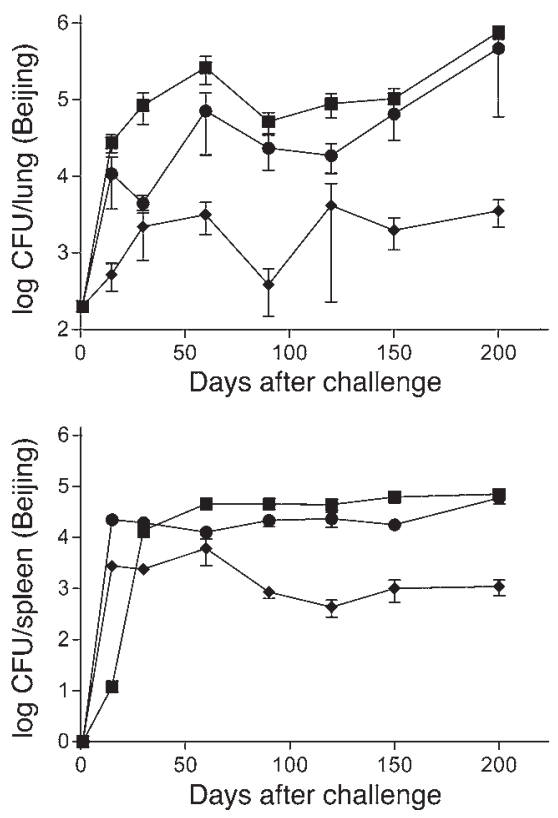
A
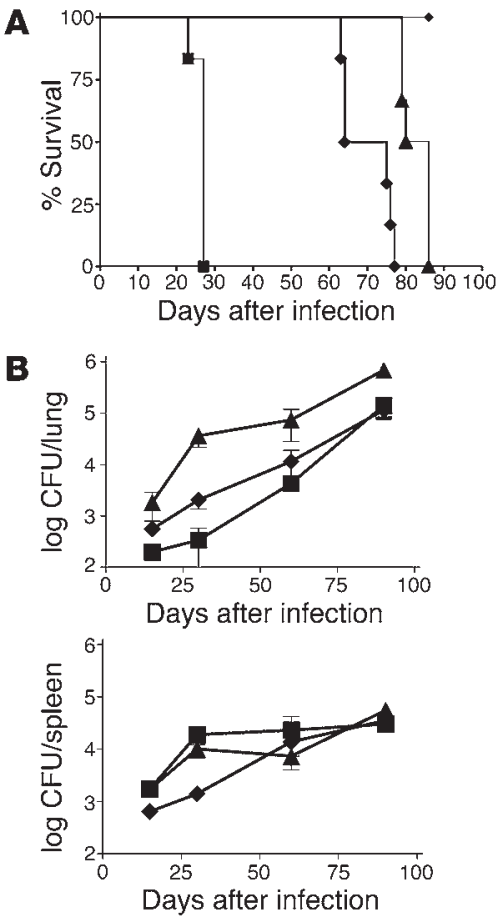

caspase cascade-inducing apoptotic cell death (11, 18-20). Apoptosis in turn results in cross-priming of mycobacterial antigens (9). Due to the immunosuppressive activities of mycobacteria (10), cross-priming would not only promote MHC I, but would also promote MHC II-restricted T cell stimulation. As shown in Figure $5, \mathrm{~A}-\mathrm{E}$, mycobacterial antigens were detected in the cytoplasm of macrophages infected with $\Delta u r e C h l y^{+}$rBCG; the antigens remained more concentrated in the phagosome of cells infected with parental BCG. Release of BCG from the phagosome of $\Delta u r e C h l y^{+}$rBCGinfected macrophages is indicated by arrows in Figure 5E. When we analyzed the density of the immunogold signal in the cytoplasm of infected cells, we found that $\Delta u r e C h l y^{+}$rBCG-infected macrophages had significantly $(P=0.026)$ more mycobacterial antigen in the cytoplasm than did macrophages infected with parental BCG (7.0 \pm 3.7 gold particles $/ \mu \mathrm{m}^{2}$ for $\Delta u r e C h l y^{+} \mathrm{rBCG}$ vs. $3.5 \pm 2.3$ par-

\section{Figure 5}

Antigen presence in the cytosol. (A-D) Bone marrow macrophages infected for 24 hours with $\triangle$ ure C hly ${ }^{+}$rBCG (A and B) and parental BCG (C and $\mathbf{D})$. The images are projections of 2 confocal planes from specimens stained for BCG (green), F-actin (red), and DNA (blue) (overlay in $\mathbf{A}$ and $\mathbf{C}$ ). Material stained with an antibody raised against $B C G$ is dispersed throughout the cytoplasm in cells infected with $\triangle u r e C$ $h l y^{+}$rBCG while BCG material is exclusively located in bacteria within cells infected with parental BCG. Scale bar in A-D: $10 \mu \mathrm{m}$. (E and F) Fine structural analysis of mycobacterial localization in bone marrow macrophages 24 hours after infection with $\triangle u r e C h l y^{+}$rBCG (E) and parental BCG $(\mathbf{F})$. Panel $\mathbf{E}$ shows strong staining of a bacterium in a phagosome; additional staining for mycobacterial material was found in the cytoplasm (indicated by an arrow). However, cytoplasmic staining was greatly reduced in cells infected with parental BCG $(\mathbf{F})$. Quantification of the density of immunogold signal in the cytoplasm of infected cells: parental BCG, $3.5 \pm 2.3$ gold particles/um²; $\Delta u r e C h / y^{+}$ rBCG, $7.0 \pm 3.7$ gold particles $/ \mu m^{2} ; P=0.022$ (Mann-Whitney $U$ test). Scale bar in E and F: $1 \mu \mathrm{m}$.

\section{Figure 4}

High safety profile of $h l y^{+}$rBCG and $\Delta u r e C h l y^{+}$rBCG vaccines. (A) SCID immune-deficient mutant mice were vaccinated i.v. with $0.5-1 \times 10^{8}$ $\mathrm{CFU}$ of $h l y^{+} \mathrm{rBCG}$ (diamonds), $\Delta u r e C h l y+$ rBCG (triangles), and parental $B C G$ (squares) to determine survival rate. $P=0.001$ for $h / y^{+}$rBCG compared with parental BCG, and $P<0.001$ for $\Delta u r e C h / y^{+}$rBCG compared with parental BCG. (B) $R A G 1^{-/-}$immunodeficient mutant mice were vaccinated i.v. with $0.5-1 \times 10^{6} \mathrm{CFU}$ of $h l y^{+} \mathrm{rBCG}$ (diamonds), $\Delta$ ureC $h / y^{+}$ rBCG (triangles), and parental BCG (squares) to determine bacterial load in lung and spleen. Differences were not statistically significant $(P=0.7$ for lung and $P=0.5$ for spleen; Mann-Whitney $U$ test). Shown is 1 representative experiment of each of 2 similar experiments.

ticles $/ \mu \mathrm{m}^{2}$ for parental BCG). Noninfected macrophages showed negligible background staining of $0.14 \pm 0.07$ particles $/ \mu \mathrm{m}^{2}$. Moreover, $\Delta$ ure $\mathrm{C} h \mathrm{ly}^{+} \mathrm{rBCG}$ induced profound apoptosis in both murine $(P<0.001)$ and human macrophages $(P<0.05)$ (Figure 6, A and $\mathrm{B}$, respectively). In contrast, parental BCG marginally increased apoptosis above background levels. The $h l y^{+}$rBCG-induced macrophage apoptosis was further increased by the absence of the ureC gene in $\Delta u r e C h l y^{+} \mathrm{rBCG}$, presumably due to the optimized $\mathrm{pH}$ for Hly activity. These results were supported by TUNEL staining of infected macrophages (Figure 6, C-E). We concluded that cytosolic MHC I loading and, to a greater extent, cross-priming contribute to improved vaccine efficacy of $b l y^{+} \mathrm{rBCG}$.

\section{Discussion}

The present report describes superior protection against tuberculosis by vaccination of mice with bly ${ }^{+}$rBCG of $L$. monocytogenes as
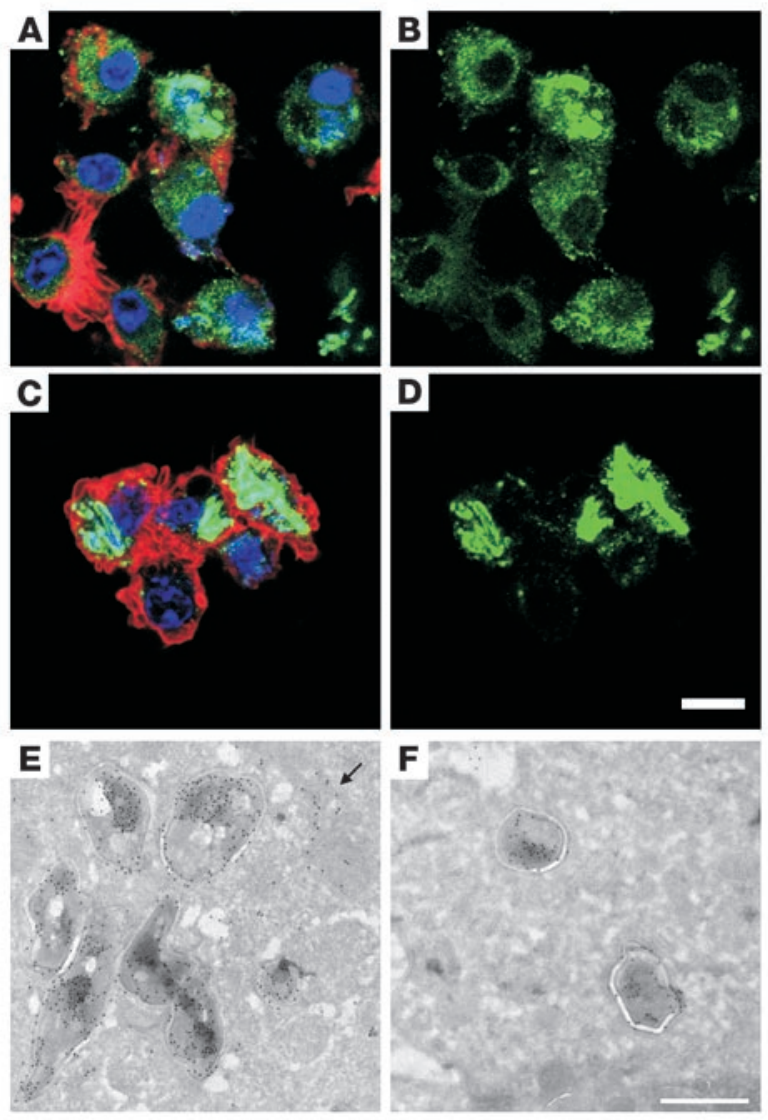

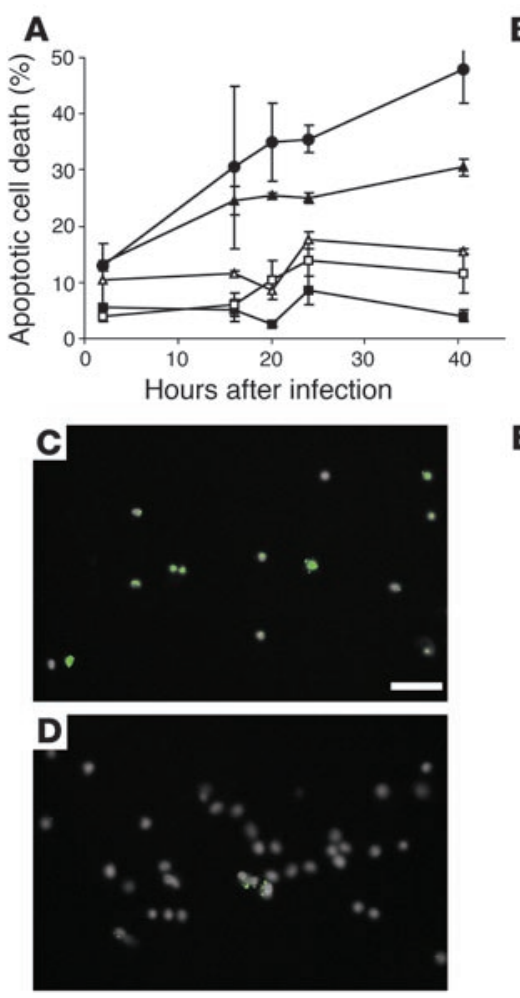

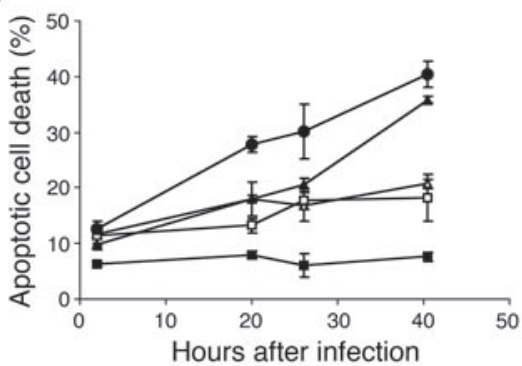

E

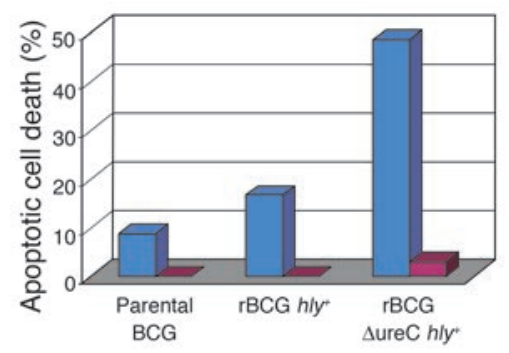

\section{Figure 6}

Increased apoptosis of infected macrophages. Primary human $(\mathbf{A})$ and murine $(\mathbf{B})$ macrophages were infected with $h l y^{+}$rBCG (filled triangles), $\Delta u r e C ~ h l y^{+}$ rBCG (circles), parental BCG (open triangles), or $\triangle$ ure $C$ rBCG (open squares), or left uninfected (filled squares). After different periods of time, apoptotic cell death was determined by measurement of DNA-fragmentation according to ref. 43. Statistical analyses resulted in values of $P<0.05$ in $\mathbf{A}$ and $P<0.001$ in B for $\Delta$ ure C hly ${ }^{+}$rBCG compared with parental BCG over the whole observation period. Shown is 1 out of 3 independent experiments each. (C-E) After 24 hours of infection with $\triangle$ ureC $h / y^{+}$rBCG (C), about $45 \%$ of bone marrow macrophages underwent apoptosis as revealed by a positive TUNEL assay while only $6 \%$ of BCG-infected macrophages (D) were apoptotic. Scale bar: $40 \mu \mathrm{m}$. (E) Quantification of the TUNEL assay: blue, BCG positive; pink, BCG negative.

low to absent protection against $M$. tuberculosis Beijing/W in BCG-vaccinated mice. In contrast, sureC $h l y^{+}$rBCG not only induced more potent protection against the laboratory strain $M$. tuberculosis $\mathrm{H} 37 \mathrm{Rv}$ but also showed greater protection against $M$. tuberculosis Beijing/W.

With respect to the mechanisms underlying improved protection by $h l y^{+}$rBCG, 2 explanations, which are not mutually exclusive, are conceivable.

compared with vaccination with parental BCG. Murine aerosolchallenge models with 2 different $M$. tuberculosis strains, the laboratory strain $\mathrm{H} 37 \mathrm{Rv}$ and a clinical isolate of the Beijing/W genotype family, revealed that $b l y^{+} \mathrm{rBCG}$ and, to a greater extent, $\Delta u r e C b_{l y}^{+}$ rBCG vaccine strains were significantly more efficacious in inducing protective immunity against tuberculosis than parental BCG.

The $\triangle$ ure $C$ rBCG strain used in our experiments was originally engineered by allelic exchange (15). ureC is involved in the maintenance of neutral intraphagosomal $\mathrm{pH}$ within $\mathrm{BCG}$-containing vacuoles (21). Hence, lack of this enzyme allows phagosomal acidification, thereby creating an optimal $\mathrm{pH}$ milieu for cytolytic functions of Hly. In addition, absence of ureC has been suggested as promoting MHC II trafficking to the macrophage cell surface (22). Hly is a major virulence factor of $L$. monocytogenes. By perforating the phagosomal membrane, it facilitates egression of L. monocytogenes into the cytosol. The acidic $\mathrm{pH}$ optimum of Hly restricts its activity in the maturing phagosome. However, BCG arrests phagosome maturation at an early stage and prohibits $\mathrm{pH}$ acidification. This obstacle of maturation arrest could be overcome, at least partially, by the removal of ureC. In the absence of ureC, the phagosomal $\mathrm{pH}$ of infected macrophages first decreases, and full activity of Hly is achieved only at optimal (acidic) pH. It is possible that the $\mathrm{pH}$ gradient subsequently breaks down due to proton influx through Hly-mediated pores. We concluded that Hly secreted by $\Delta$ ureC $h l y^{+}$ rBCG was more active than that secreted by $h l y^{+}$rBCG because of an optimized phagosomal $\mathrm{pH}$ milieu for Hly.

BCG vaccination protects newborns against conventional $M$. tuberculosis isolates $(1,7,23,24)$ but has apparently lost vaccine efficacy against newly emerging isolates, notably of the $M$. tuberculosis Beijing/W genotype family (3, 24-28). In experimental animal models, BCG confers only weak protection against the clinical isolate Beijing/W (29). Consistent with this observation, we found
First, the bly ${ }^{+}$rBCG vaccine strains may have enhanced cytosolic antigen loading of MHC I molecules in infected APCs to prime CD8 $\mathrm{T}$ cells (17). Consistent with this notion, we detected a higher abundance of mycobacterial antigens in the cytoplasm of macrophages infected with $\triangle u r e C h l y^{+}$rBCG as compared with those infected with parental BCG. Second, perforation of the phagosomal membrane by Hly may promote cross-priming by induction of apoptosis within the infected macrophage (9). It remains to be clarified whether $M$. tuberculosis preferentially induces or inhibits apoptosis $(9,30,31)$, and it is likely that the outcome of apoptosis regulation depends on specific conditions. Additionally, we assume that $\Delta u r e C$ $b l y^{+}$rBCG induces apoptosis via a different mechanism than does M. tuberculosis. Hly from L. monocytogenes has been shown to induce apoptotic cell death in different cell types $(19,20,32)$. It is likely that membrane perforation by Hly secreted by L. monocytogenes or by $\Delta$ ure $C h l y^{+}$rBCG facilitates delivery of phagolysosomal proteases into the cytosol (32), where cathepsins have been shown to induce apoptosis via the mitochondrial pathway (18). Although first experiments with a macrophage cell line did not reveal cytolytic activity of ly $^{+} \mathrm{rBCG}^{+}$by lactate dehydrogenase-release assay (33), we found significant induction of apoptotic cell death by measuring DNAfragmentation of primary macrophages. Apoptosis of infected cells promotes cross-priming of mycobacterial antigens via uptake of apoptotic vesicles by DCs and therefore improves cell-mediated immune responses (9). Transfer of antigens from infected cells to potent APCs such as DCs may represent an alternative pathway involving infection-mediated apoptosis of host cells, by which CD8 $T$ cells recognize antigens of the phagosomally secluded tubercle bacillus (9). Recent experiments demonstrated that vesicles containing mycobacterial antigens derived from macrophages infected with BCG are capable of adoptively transferring protection against M. tuberculosis to mice (our unpublished observations). 
In sum, we assume that improved vaccine efficacy of $\Delta u r e C$ $h l y^{+}$rBCG as compared with bly ${ }^{+}$rBCG and parental BCG is best explained by efficient perforation of phagosomal membranes by Hly. Hly meets an acidic pH optimum in the phagosome due to absent ureC activity. Perforation promotes antigen translocation into the cytoplasm and facilitates cross-priming through increased apoptosis. We consider the latter mechanism of major importance for augmented vaccine efficacy of $\Delta u r e C h l y^{+}$rBCG.

Concerning in vivo safety of the $\Delta u r e C h l y^{+} \mathrm{rBCG}$ and $b l y^{+} \mathrm{rBCG}$ vaccines, we observed even lower virulence as compared with that of parental BCG in immunodeficient SCID mice, which is most likely due to reduced intracellular persistence of both $\mathrm{rBCG}$ strains in infected host cells $(34,35)$. Consistent with our finding that $\Delta u r e C h l y^{+}$rBCG was highly attenuated in SCID mice, $\Delta u r e C$ rBCG has already shown a slight decrease in multiplication and persistence in the lung of vaccinated mice compared with parental BCG (36). This is in agreement with the observation that ureC-deficient BCG is significantly attenuated in its capacity to block MHC class II expression (22). Both for safety and for vaccine efficacy reasons, we selected $\Delta u r e C h l y^{+}$rBCG for good manufacturing practices (GMP) production and clinical trials after licensing to Vakzine Projekt Management GmbH (Hannover, Germany).

A strategy to improve antigen display by BCG has previously been reported (37). Increased vaccine efficacy of the $2 \mathrm{rBCG}$ strains is based on different mechanisms - elevated antigen secretion by the rBCG strain of Horwitz et al. (37) and altered intracellular behavior of the rBCG strain described here. A combination of both strains, therefore, may be considered for replacing conventional BCG for vaccination of newborns. Recently, several subunit vaccine candidates have been described, which can be used to boost immune responses induced by BCG prime (38-42). It is tempting to assume that a combination of priming with improved BCG such as $\Delta u r e C$ $h y^{+}$rBCG and boosting with the most efficacious subunit vaccine would provide more powerful intervention measures against one of the major threats among infectious diseases, tuberculosis.

\section{Methods}

Mycobacterial strains and cell lines. The BCG strains Danish 1331 (Statens Serum Institut, Copenhagen, Denmark) and BCG Pasteur 1173P2 (originally provided by B. Gicquel, Institut Pasteur, Paris, France) were cultured in Dubos broth base (Difco; BD Diagnostics) supplemented with Dubos medium albumin (Difco; BD Diagnostics) at $37^{\circ} \mathrm{C}$. A midlogarithmic culture was aliquoted and stored at $-70^{\circ} \mathrm{C}$ until use. The mycobacteria-Escherichia coli shuttle vector $P M V 306$ was used to integrate the bly gene into the mycobacterial chromosome of BCG Danish 1331, which resulted in the respective Hly recombinant strain, $h_{y}{ }^{+} \mathrm{rBCG}^{+}$Danish (35). To generate $\Delta$ ureC $b y^{+} \mathrm{rBCG}$, electrocompetent $M$. bovis BCG Pasteur $\Delta u r e C: a p h(15)$ was transformed using the chromosomal integrative shuttle vector pMV306hyg-Hly, and hygromycin-resistant clones were selected. Plasmid pMV306hyg-Hly was obtained by inserting the hygromycin resistance gene from pMV206-hyg into pMV306Hly (35) to replace the kanamycin-resistance marker. M. tuberculosis H37Rv (originally obtained from J.K. Seydel, Forschungsinstitut Borstel, Borstel, Germany) and M. tuberculosis Beijing/W (RIVM No. 17919, country of isolation: Mongolia) were passaged in mice and grown in Dubos broth base supplemented with $10 \%$ Dubos medium albumin (Difco; BD Diagnostics), then stored in aliquots at $-70^{\circ} \mathrm{C}$. P815 mastocytoma cells and murine macrophage-like cells J774A.1, both of H-2 ${ }^{\mathrm{d}}$ haplotype, were obtained from ATCC and cultured in RPMI 1640 (Life Technologies Inc.) supplemented with 10\% FCS, penicillin (100 IU/ml), streptomycin (100 IU/ml), and 2-methoxy-1ethanol. This medium is referred to as RP10 medium.
All animal experiments were conducted with the approval of the Landesamt für Arbeitsschutz, Gesundheitsschutz und technische Sicherheit (Berlin, Germany). BALB/c mice were bred at the central animal facilities of the Max Planck Institute for Infection Biology at the Bundesinstitut für gesundheitlichen Verbraucherschutz und Veterinärmedizin (Berlin, Germany). Animals were kept under specific pathogen-free conditions and fed autoclaved food and water ad libitum.

RT-PCR. Total RNA from BCG was isolated using TRIzol RNA isolation kit (Gibco BRL; Invitrogen Corp.). The quality, purity, and concentration of the RNA was determined by Bioanalyzer (Agilent). RNA ( $1 \mu \mathrm{g})$ was used for RT-PCR using Access Quick RT-PCR System (Promega). PCR was carried out for 30 cycles $\left(94^{\circ} \mathrm{C}\right.$ for 1 minute; $57^{\circ} \mathrm{C}$ for 1 minute; $72^{\circ} \mathrm{C}$ for 1.5 minutes) with the following primers (MWG Biotech): forward-primer: $5^{\prime}$-AAGGGGCACAGGTATGAAAG-3'; and reverse-primer: 5'-GATTTCATCCGCGTGTTTCT-3'. PCR products were analyzed by agarose gel electrophoresis.

In vitro hemolysis assay. One $\mathrm{ml}$ of a $50 \%$ sheep erythrocytes solution in Alsever buffer (Fiebig-Nährstofftechnik) was incubated with each $2 \times 10^{6}$ CFU of $h l y^{+}$rBCG, $\triangle$ ureC $h l y^{+}$rBCG, and parental BCG as well as with $L$. monocytogenes as control at $37^{\circ} \mathrm{C}$ in a water bath under constant shaking. Every 60 minutes, the absorption at $410 \mathrm{~nm}$ was measured in order to determine the amount of free hemoglobin.

Phagosomal pH measurements. Bone marrow macrophages or RAW 264.7 murine macrophage cells were grown on glass slides at $37^{\circ} \mathrm{C}$ and infected with the different BCG strains for various time periods as indicated in the figure legends. To identify lysosomes that contained bacteria, the BCG were stained with DID (Vibrant Cell Labeling Kit; Invitrogen Corp.). LysoSensor Yellow/Blue (Invitrogen Corp.) was added to the medium at a final concentration of $15 \mu \mathrm{M}$. This dye is spontaneously taken up by the cells into lysosomes and phagosomes. Fluorescence microscopic experiments were performed with a Leica DMRB microscope equipped with a Leica Pl Fluo$\operatorname{tar} 40 \times / 1.00-0.50$ oil, a Nikon DYM 1200 digital camera, and a $100 \mathrm{~W}$ HBO lamp (Osram). For LysoSensor green fluorescence detection, the filter set was composed of exciter $380 / 13 \mathrm{~nm}$, dichroic $400 \mathrm{~nm}$, and emitter $560 / 80 \mathrm{~nm}$, while for blue fluorescence the combination was exciter $360 / 40 \mathrm{~nm}$, dichroic $400 \mathrm{~nm}$, and emitter 360/40 nm. DID fluorescence was detected with a regular filter set for dark red dyes. For ratiometric analysis, micrographs of dye-labeled phagosomes containing bacteria were recorded. Digital images were processed using ImageJ software version 1.33a (NIH; http://rsb.info.nih.gov/ij). The green and blue channels were extracted from the RGB (red, green, blue) images, and the fluorescence intensity over lysosomes was measured. The ratio of blue to green fluorescence of the lysosomes was calculated according to the following formula: intensity (resulting image) $=25 \times$ intensity (blue)/intensity (green). The resulting images were converted to pseudocolor to indicate $\mathrm{pH}$ values. To obtain a pH calibration curve, cells were stained with LysoSensor Yellow/ Blue coupled to dextran (Invitrogen Corp.), treated with $10-\mu \mathrm{M}$ monensin and $10-\mu \mathrm{M}$ nigericin, and equilibrated for 2 minutes with calibrated PBS buffer solutions ( $\mathrm{pH}$ from 4.0 to 7.5 ) prior to image acquisition. In another set of experiments, parental BCG and $\Delta u r e C h l y^{+}$rBCG were covalently coupled to the $\mathrm{pH}$-sensitive dye Cypher 5 (Amersham Biosciences). This dye emits dark red fluorescence that becomes more intense upon acidification. Cypher 5-coupled bacteria were used to infect murine bone marrow macrophages, and changes in fluorescence intensity over 60 minutes after infection were recorded on a Zeiss Axiovert 200M microscope equipped with a Cy5 filter set on a Hamamatsu Orca digital camera.

Determination of apoptosis. Apoptotic cell death was determined according to the method of Nicoletti et al. (43). Briefly, cells were harvested and centrifuged in a minifuge (Heraeus) at 3,600 $\mathrm{g}$ for 5 minutes, washed once with PBS, and subsequently resuspended in a buffer containing $0.1 \%$ (w/v) sodium citrate, $0.1 \%(\mathrm{v} / \mathrm{v})$ Triton X-100, and $50 \mu \mathrm{g} / \mathrm{ml}$ propidium 
iodide (Sigma-Aldrich). After incubation at $4^{\circ} \mathrm{C}$ in the dark for at least 16 hours, apoptotic nuclei were quantified by FACScan (BD). For detecting apoptotic nuclei by the TUNEL method, bone marrow macrophages were seeded on glass coverslips, infected with the various BCG strains for 8 and 24 hours, and fixed. The TUNEL assay kit (In Situ Cell Death Detection Kit; Roche Diagnostics Corp.) was employed following the instructions of the manufacturer. The signal of the FITC-labeled nucleotides was intensified using an anti-FITC antibody coupled to Alexa Fluor 488 (Invitrogen Corp.). Nuclei were counterstained with Bisbenzimide (Hoechst 33258), and the percentage of TUNEL-positive cells was determined using Image software version 1.33a.

Confocal microscopy. Bone marrow macrophages grown on coverslips were infected with BCG strains for the indicated periods, fixed in $4 \%$ paraformaldehyde, permeabilized in $1 \%$ Triton X-100 in PBS, blocked, and stained with an antibody raised against BCG. To indicate localization in the cells, F-actin was stained with phalloidin and DNA with Draq5 (Biostatus Ltd.). Samples were embedded in Mowiol (Sigma-Aldrich) and analyzed with a Leica TCS-SP confocal microscope.

Cryoelectron microscopy. Bone marrow macrophages were infected with BCG strains. Infected cultures were fixed with $4 \%$ PFA/PBS and harvested using a rubber policeman. Suspended cells were transferred to a centrifuge tube and pelleted at $1000 \mathrm{~g}$ for 2 minutes. The supernatant was discarded and the cells were carefully suspended in $30 \mu \mathrm{l}$ of melted low melting point agarose (2\% PBS; Fluka). Droplets of the suspension were placed on Parafilm (American National Can Company) in a humidified chamber and left to gel on ice. After gelling, droplets were cut to cubes with an edge length of about $1 \mathrm{~mm}$. The cubes were gradually infiltrated in a mixture of $1.6 \mathrm{M}$ saccharose $/ 25 \%(\mathrm{w} / \mathrm{v})$ polyvinylpyrrolidone in PBS. Infiltrated cubes were mounted on aluminium stubs, snap frozen in liquid nitrogen, and transferred to an RMC ultramicrotome equipped with a cryo chamber. The specimens were trimmed using a diamond trimming device and then cut to ultrathin cryosections. The sections were collected on Formvar-coated nickel grids (Plano) and incubated on blocking buffer (1\% BSA, 5\% NGS, $10 \%$ cold water fish gelatin in PBS). The anti-BCG rabbit serum was diluted to $1: 1,000$ in the same buffer and reacted with the specimens for 1 hour at $37^{\circ} \mathrm{C}$ followed by addition of a goat anti-rabbit serum conjugated to 12 $\mathrm{nm}$ gold colloids. As controls, noninfected macrophages with anti-BCG serum and nonimmune rabbit serum were used. Sections were analyzed in a Leo 906E transmission electron microscope (Leo/Zeiss). Statistical analysis on cytoplasmic staining intensity was performed using ImageJ software version 1.33a. Twenty random images (magnification, $\times 12,930$ ) of murine bone marrow macrophages infected with the BCG strains or not infected were analyzed for immunogold localization not linked to phagosomes. Staining intensity was expressed as gold particles per $\mu \mathrm{m}^{2}$.
Vaccination and challenge of mice. Mice were immunized i.v. with $10^{6} \mathrm{CFU}$ of BCG strains. Immunization via the i.v. route has been shown to result in the same level of protection against $M$. tuberculosis challenge as immunization via the s.c. route (44). At 120 days after vaccination, animals were aerosol challenged with 100-200 CFU of M. tuberculosis H37Rv or Beijing/W per lung using an aerosol chamber (Glas-Col) as described (42). The inoculum was confirmed at day 1 after infection by plating the complete lungs and spleens of mice onto Middlebrook 7H11/ampicillin plates (Becton Dickinson).

Statistical analyses. Statistical analyses were performed using Mann-Whitney $U$ test (CFU assays), Friedman test (CFU time courses), log-rank test (survival assays), and 1-way ANOVA for repeated measurements (apoptosis assays). Differences were considered significant at $P<0.05$.

\section{Acknowledgments}

The authors thank Beatrix Fauler for immunostainings of ultrathin cryosections and Silke Bandermann for excellent technical assistance. The BCG Pasteur 1173P2 strain was kindly provided by B. Gicquel. We thank D. Russell for providing unpublished data on the impact of ureC on $\mathrm{pH}$ neutralization. The paper received financial support from European Union FP5 (QLK21999-01093) and FP6 (LSHP-CT-2003-503367) as well as from the German Science Foundation Priority Programme Novel Vaccination Strategies (SPP 1089, KA 573/4-2).

Received for publication January 31, 2005, and accepted in revised form June 7, 2005.

Address correspondence to: Stefan H.E. Kaufmann, Max Planck Institute for Infection Biology, Department of Immunology, Schumannstrasse 21/22, D-10117 Berlin, Germany. Phone: 4930-28460-500 or 49-30-28460-506; Fax: 49-30-28460-501; E-mail: Kaufmann@mpiib-Berlin.mpg.de.

Leander Grode's present address is: Vakzine-Projekt Management $\mathrm{GmbH}$, Braunschweig, Germany.

Peter Seiler's present address is: Bayer HealthCare AG, Wuppertal, Germany.

Jürgen Hess's present address is: responsif GmbH, Erlangen, Germany.

Leander Grode, Peter Seiler, and Sven Baumann contributed equally to this work.
1. Fine, P.E. 2001. BCG: the challenge continues [review]. Scand. J. Infect. Dis. 33:243-245.

2. Fine, P.E.M., Carneiro, I.A.M., Milstien, J.B., and Clements, M. 1999. Issues relating to the use of BCG in immunisation programmes. A discussion document. WHO. Geneva, Switzerland. WHO/ V\&B/99.23. http://www.who.int/vaccines-documents/DocsPDF99/www9943.pdf.

3. Glynn, J.R., Whiteley, J., Bifani, P.J., Kremer, K., and van Soolingen, D. 2002. Worldwide occurrence of Beijing/W strains of Mycobacterium tuberculosis: a systematic review. Emerg. Infect. Dis. 8:843-849.

4. Manca, C., et al. 2004. Differential monocyte activation underlies strain-specific Mycobacterium tuberculosis pathogenesis. Infect. Immun. 72:5511-5514.

5. Reed, M.B., et al. 2004. A glycolipid of hypervirulent tuberculosis strains that inhibits the innate immune response. Nature. 431:84-87.

6. Munsiff, S.S., et al. 2003. Persistence of a highly resistant strain of tuberculosis in New York City during 1990-1999. J. Infect. Dis. 188:356-363.

7. Espinal, M.A. 2003. The global situation of MDRTB [review]. Tuberculosis (Edinb). 83:44-51.

8. Kaufmann, S.H.E., and McKnight, A.J. 2005 Annulling a dangerous liaison: vaccination strategies against AIDS and tuberculosis. Nat. Med. 11(4 Suppl.):S33-S44.

9. Schaible, U.E., et al. 2003. Apoptosis facilitates antigen presentation to T lymphocytes through MHC-I and CD1 in tuberculosis. Nat. Med. 9:1039-1046.

10. Pancholi, P., Mirza, A., Bhardwaj, N., and Steinman, R.M. 1993. Sequestration from immune Cd4+ T-Cells of mycobacteria growing in human macrophages. Science. 260:984-986.

11. Mandal, M., and Lee, K.D. 2002. Listeriolysin O-liposome-mediated cytosolic delivery of macromolecule antigen in vivo: enhancement of antigen-specific cytotoxic T lymphocyte frequency, activity, and tumor protection. Biochim Biophys Acta. 1563:7-17.
12. Portnoy, D.A., Chakraborty, T., Goebel, W., and Cossart, P. 1992. Molecular determinants of Listeria monocytogenes pathogenesis [review]. Infect. Immun. 60:1263-1267.

13. Glomski, I.J., Gedde, M.M., Tsang, A.W., Swanson, J.A., and Portnoy, D.A. 2002. The Listeria monocytogenes hemolysin has an acidic $\mathrm{pH}$ optimum to compartmentalize activity and prevent damage to infected host cells. J. Cell Biol. 156:1029-1038.

14. Honer zu Bentrup, K., and Russell, D.G. 2001. Mycobacterial persistence: adaptation to a changing environment [review]. Trends Microbiol. 9:597-605.

15. Reyrat, J.M., Berthet, F.X., and Gicquel, B. 1995. The urease locus of Mycobacterium tuberculosis and its utilization for the demonstration of allelic exchange in Mycobacterium bovis bacillus Calmette-Guerin. Proc. Natl. Acad. Sci. U. S. A. 92:8768-8772.

16. Schaible, U.E., Sturgill-Koszycki, S., Schlesinger, P.H., and Russell, D.G. 1998. Cytokine activation 
leads to acidification and increases maturation of Mycobacterium avium-containing phagosomes in murine macrophages. J. Immunol. 160:1290-1296.

17. Kaufmann, S.H.E. 1993. Immunity to intracellular bacteria. Annu. Rev. Immunol. 11:129-163.

18. Cirman, T., et al. 2004. Selective disruption of lysosomes in HeLa cells triggers apoptosis mediated by cleavage of bid by multiple papain-like lysosomal cathepsins. J. Biol. Chem. 279:3578-3587.

19. Rogers, H.W., Callery, M.P., Deck, B., and Unanue, E.R. 1996. Listeria monocytogenes induces apoptosis of infected hepatocytes. J. Immunol. 156:679-684.

20. Guzman, C.A., et al. 1996. Apoptosis of mouse dendritic cells is triggered by listeriolysin, the major virulence determinant of Listeria monocytogenes. Mol. Microbiol. 20:119-126.

21. Clemens, D.L., Lee, B.Y., and Horwitz, M.A. 1995. Purification, characterization, and genetic-analysis of Mycobacterium tuberculosis urease, a potentially critical determinant of host-pathogen interaction. J. Bacteriol. 177:5644-5652.

22. Sendide, K., Deghmane, A.E., Reyrat, J.M., Talal, A., and Hmama, Z. 2004. Mycobacterium bovis BCG urease attenuates major histocompatibility complex class II trafficking to the macrophage cell surface. Infect. Immun. 72:4200-4209.

23. Bifani, P.J., Mathema, B., Kurepina, N.E., and Kreiswirth, B.N. 2002. Global dissemination of the Mycobacterium tuberculosis W-Beijing family strains. Trends Microbiol. 10:45-52.

24. Filliol, I., et al. 2002. Global distribution of Mycobacterium tuberculosis spoligotypes. Emerg. Infect. Dis. 8: $1347-1349$.

25. van Soolingen, D. 2001. Molecular epidemiology of tuberculosis and other mycobacterial infections: main methodologies and achievements [review]. J. Intern. Med. 249:1-26.

26. Rad, M.E., et al. 2003. Mutations in putative mutator genes of Mycobacterium tuberculosis strains of the W-Beijing family. Emerg. Infect. Dis. 9:838-845.

27. Toungoussova, O.S., Mariandyshev, A., Bjune, G.,
Sandven, P., and Caugant, D.A. 2003. Molecular epidemiology and drug resistance of Mycobacterium tuberculosis isolates in the archangel prison in Russia: predominance of the W-Beijing clone family. Clin. Infect. Dis. 37:665-672.

28. van Soolingen, D., et al. 1995. Predominance of a single genotype of Mycobacterium tuberculosis in countries of east Asia. J. Clin. Microbiol. 33:3234-3238.

29. Lopez, B., et al. 2003. A marked difference in pathogenesis and immune response induced by different Mycobacterium tuberculosis genotypes. Clin. Exp. Immunol. 133:30-37.

30. Fayyazi, A., et al. 2000. Apoptosis of macrophages and $T$ cells in tuberculosis associated caseous necrosis. J. Pathol. 191:417-425.

31. Keane, J., Remold, H.G., and Kornfeld, H. 2000. Virulent Mycobacterium tuberculosis strains evade apoptosis of infected alveolar macrophages. J. Immunol. 164:2016-2020.

32. Lee, K.D., Oh, Y.K., Portnoy, D.A., and Swanson, J.A. 1996. Delivery of macromolecules into cytosol using liposomes containing hemolysin from Listeria monocytogenes. J. Biol. Chem. 271:7249-7252.

33. Hess, J., et al. 1998. Mycobacterium bovis bacille Calmette-Guerin strains secreting listeriolysin of Listeria monocytogenes. Proc. Natl. Acad. Sci. U. S. A. 95:5299-5304.

34. Rossi, L., et al. 2004. Effect of listeriolysin O-loaded erythrocytes on Mycobacterium avium replication within macrophages. J. Antimicrob. Chemother. 53:863-866

35. Hess, J., et al. 1998. Mycobacterium bovis bacille Calmette-Guerin strains secreting listeriolysin of Listeria monocytogenes. Proc. Natl. Acad. Sci.U.S. A. 95:5299-5304

36. Reyrat, J.M., Lopez-Ramirez, G., Ofredo, C., Gicquel, B., and Winter, N. 1996. Urease activity does not contribute dramatically to persistence of Mycobacterium bovis Bacillus Calmette-Guerin. Infect. Immun. 64:3934-3936.
37. Horwitz, M.A., Harth, G., Dillon, B.J., and MaslesaGalic, S. 2000. Recombinant bacillus CalmetteGuerin (BCG) vaccines expressing the Mycobacterium tuberculosis $30-\mathrm{kDa}$ major secretory protein induce greater protective immunity against tuberculosis than conventional BCG vaccines in a highly susceptible animal model. Proc. Natl. Acad. Sci. U. S. A. 97:13853-13858.

38. McShane, H., et al. 2004. Recombinant modified vaccinia virus Ankara expressing antigen $85 \mathrm{~A}$ boosts BCG-primed and naturally acquired antimycobacterial immunity in humans. Nat. Med. 10:1240-1244.

39. Brooks, J.V., Frank, A.A., Keen, M.A., Bellisle, J.T., and Orme, I.M. 2001. Boosting vaccine for tuberculosis. Infect. Immun. 69:2714-2717.

40. Vordermeier, H.M., et al. 2004. Cellular immune responses induced in cattle by heterologous primeboost vaccination using recombinant viruses and bacille Calmette-Guerin. Immunology. 112:461-470.

41. Goonetilleke, N.P., et al. 2003. Enhanced immunogenicity and protective efficacy against Mycobacterium tuberculosis of bacille Calmette-Guerin vaccine using mucosal administration and boosting with a recombinant modified vaccinia virus Ankara. J. Immunol. 171:1602-1609.

42. Mollenkopf, H.J., et al. 2004. Application of mycobacterial proteomics to vaccine design: improved protection by Mycobacterium bovis BCG primeRv3407 DNA boost vaccination against tuberculosis. Infect. Immun. 72:6471-6479.

43. Nicoletti, I., Migliorati, G., Pagliacci, M.C., Grignani, F., and Riccardi, C. 1991. A rapid and simple method for measuring thymocyte apoptosis by propidium iodide staining and flow-cytometry. J. Immunol. Methods. 139:271-279.

44. Palendira, U., Bean, A.G.D., Feng, C.G., and Britton, W.J. 2002. Lymphocyte recruitment and protective efficacy against pulmonary mycobacterial infection are independent of the route of prior Mycobacterium bovis BCG immunization. Infect. Immun. 70:1410-1416 